\title{
Abnormal Brain Responses to Action Observation in Complex Regional Pain Syndrome
}

\section{Hotta, Jaakko}

2017-03

Hotta , J , Saari , J , Koskinen, M , Hlushchuk, Y , Forss , N \& Hari , R 2017 , ' Abnormal Brain Responses to Action Observation in Complex Regional Pain Syndrome ', Journal of Pain , vol. 18 , no. 3 , pp. 255-265 . https://doi.org/10.1016/j.jpain.2016.10.017

http://hdl.handle.net/10138/234475

https://doi.org/10.1016/j.jpain.2016.10.017

publishedVersion

Downloaded from Helda, University of Helsinki institutional repository.

This is an electronic reprint of the original article.

This reprint may differ from the original in pagination and typographic detail.

Please cite the original version. 


\title{
Abnormal Brain Responses to Action Observation in Complex Regional Pain Syndrome
}

\author{
Jaakko Hotta, ${ }^{*}, \dagger, \ddagger$ Jukka Saari, ${ }^{*}$ Miika Koskinen, ${ }^{*}, \S$ Yevhen Hlushchuk, ${ }^{*}, \pi$ Nina Forss,, , \\ and Riitta Hari*, ${ }^{*}$ \\ *Systems and Clinical Neuroscience, Department of Neuroscience and Biomedical Engineering, ${ }^{\dagger}$ Aalto Neurolmaging, \\ Aalto University School of Science, Espoo, Finland. \\ ${ }^{\ddagger}$ Department of Neurology, Helsinki University Hospital, and Clinical Neurosciences, Neurology, University of Helsinki, \\ Finland. \\ ${ }^{\S}$ Department of Physiology, Faculty of Medicine, University of Helsinki, Helsinki, Finland. \\ "HUS Medical Imaging Center, Radiology, University of Helsinki and Helsinki University Hospital, Helsinki, Finland. \\ "Department of Art, Aalto University School of Arts, Design and Architecture, Helsinki, Finland.
}

\begin{abstract}
Patients with complex regional pain syndrome (CRPS) display various abnormalities in central motor function, and their pain is intensified when they perform or just observe motor actions. In this study, we examined the abnormalities of brain responses to action observation in CRPS. We analyzed 3-T functional magnetic resonance images from 13 upper limb CRPS patients (all female, ages 31-58 years) and 13 healthy, age- and sex-matched control subjects. The functional magnetic resonance imaging data were acquired while the subjects viewed brief videos of hand actions shown in the first-person perspective. A pattern-classification analysis was applied to characterize brain areas where the activation pattern differed between CRPS patients and healthy subjects. Brain areas with statistically significant group differences $(q<.05$, false discovery rate-corrected) included the hand representation area in the sensorimotor cortex, inferior frontal gyrus, secondary somatosensory cortex, inferior parietal lobule, orbitofrontal cortex, and thalamus. Our findings indicate that CRPS impairs action observation by affecting brain areas related to pain processing and motor control. Perspective: This article shows that in CRPS, the observation of others' motor actions induces abnormal neural activity in brain areas essential for sensorimotor functions and pain. These results build the cerebral basis for action-observation impairments in CRPS.
\end{abstract}

(c) 2016 by the American Pain Society

Key words: Action observation, chronic pain, CRPS, fMRI, multivoxel pattern analysis.

$\mathrm{C}$ omplex regional pain syndrome (CRPS) manifests with pain, hyperesthesia, allodynia, edema, and motor, vasomotor, and sudomotor symptoms in a

\footnotetext{
Received March 10, 2016; Revised August 30, 2016; Accepted October 28, 2016.

J.H. and J.S. contributed equally to this work.

This study was supported by the Academy of Finland (grants 131483 and 263800 to R.H.), by the European Research Council (Advanced Grant 232946 to R.H.), by the Strategic Centre for Science, Technology and Innovation in Health and Well-being (SHOK; SalWe Ltd, Finland to N.F. and R.H.), by the Emil Aaltonen Foundation (to J.H.), by the Jane and Aatos Erkko foundation (M.K.), and by the Aalto Brain Centre (M.K.).

The authors have no conflicts of interest to declare.

Supplementary data accompanying this article are available online at www.jpain.org and www.sciencedirect.com.

Address reprint requests to Jaakko Hotta, MD, Department of Neuroscience and Biomedical Engineering, PO Box 13000, Aalto 00076, Finland. E-mail: jaakko.hotta@aalto.fi

$1526-5900 / \$ 36.00$

(C) 2016 by the American Pain Society

http://dx.doi.org/10.1016/j.jpain.2016.10.017
}

limb. It is a disabling disorder without effective treatment. Because the pathophysiology is incompletely understood and definitive biomarkers are lacking, the diagnosis is based on a set of symptoms and signs ${ }^{27-29}$ and thus also faced with criticism for being unspecific. ${ }^{4,14,43,50,53}$ More thorough understanding of pathophysiological mechanisms is needed for development of biomarkers and eventually better treatments for CRPS.

The current concept of CRPS pathophysiology is multifaceted. In addition to peripheral mechanisms (inflammation and vasomotor dysfunction), the central nervous system is essentially involved in the disease process. ${ }^{6,44}$

The central contribution is manifested especially in the peculiar motor characteristics of CRPS. For example, patients need exaggerated effort to perform movements with the painful limb ${ }^{23}$ and even imagined movements 
can be abnormally slow. ${ }^{57}$ The movement-related pain and swelling are decreased when patients see their limb in a smaller size, whereas these symptoms increase when the limb image is magnified. ${ }^{46}$ If the patients do not see the limb, the sense of position ${ }^{38}$ and the performance of simple motor tasks ${ }^{22}$ are impaired. It has been suggested that such symptoms can discriminate CRPS from other chronic pain disorders. ${ }^{22,27,29}$ The central mechanisms underscoring these manifestations can be assessed using modern functional neuroimaging.

Intriguingly, not only movements, ${ }^{15,64}$ but also motor imagery, ${ }^{47}$ or just observing motor actions ${ }^{31}$ aggravate pain in CRPS. In healthy persons, action observation is known to affect brain areas that support the actual performance of such actions (for a review of motor mirroring, see Rizzolatti and Fogassi ${ }^{54}$ ). Because the brain's motor circuitry is activated abnormally in CRPS patients, ${ }^{40}$ brain responses to action observation might expose novel pathophysiology in CRPS

We thus performed functional magnetic resonance (MR) imaging ( $\mathrm{fMRI}$ ) on upper limb CRPS type 1 patients and a control group of healthy adults who viewed hand actions in videos. To effectively reveal abnormalities of brain responses to action observation, we applied pattern classification analysis (searchlight classification). ${ }^{36}$ This approach provides detailed information about brain sites where responses to action observation differ between patients and control subjects.

\section{Methods}

\section{Subjects}

We identified 96 patients with CRPS from the patient registry of the Pain Clinic at the Helsinki University Hospital. In addition, nearby hospitals referred to us 19 patients to be included in the study. From this pool of patients, we found 17 right-handed upper limb CRPS type 1 patients (16 female, 1 male), who met our study criteria given in Fig $1 \mathrm{~A}$; the gender imbalance likely reflects the 3 to 4 times higher incidence of CRPS in female than in male individuals. ${ }^{16,55}$ The patient selection process is described in more detail in Fig 1B. The control group comprised 20 right-handed healthy adults (19 female, 1 male) recruited primarily using e-mail advertisements. From this subject sample, 1 healthy control subject was excluded because of an incidental exclusive MR abnormality. As an additional inclusion criterion, we required alertness during the recording: on the basis of continuous online eye-tracking during the whole MR scanning, 13 patients $(76.5 \%)$ and 15 healthy control subjects $(78.9 \%)$ were sufficiently alert to be included in the analysis (for details, see the Supplementary Text). Finally, to match the group size for classification, we selected 13 alert control subjects, optimized to match the patients for sex and age, as well as for the MR scanner used (because of a scanner change in the middle of the study). The recruitment and MR scanning took place between January 1, 2011 and January 30, 2013.

\section{A}

Inclusion criteria:

1. Diagnosis of upper limb CRPS type 1 (the Budapest criteria)

2. Disease duration more than 6 months

3. Severe rest or movement pain*

4. Right-handedness

5. Age $18-65$ years

*more than 4 on a scale from 0 (= no pain) to 10 (= extreme pain)

Exclusion criteria:

1. Other major neurological or psychiatric diagnosis

2. Drug or alcohol addiction

3. Contra-indication for MRI (e.g. metal in the body)

\section{B}

Patient selection process

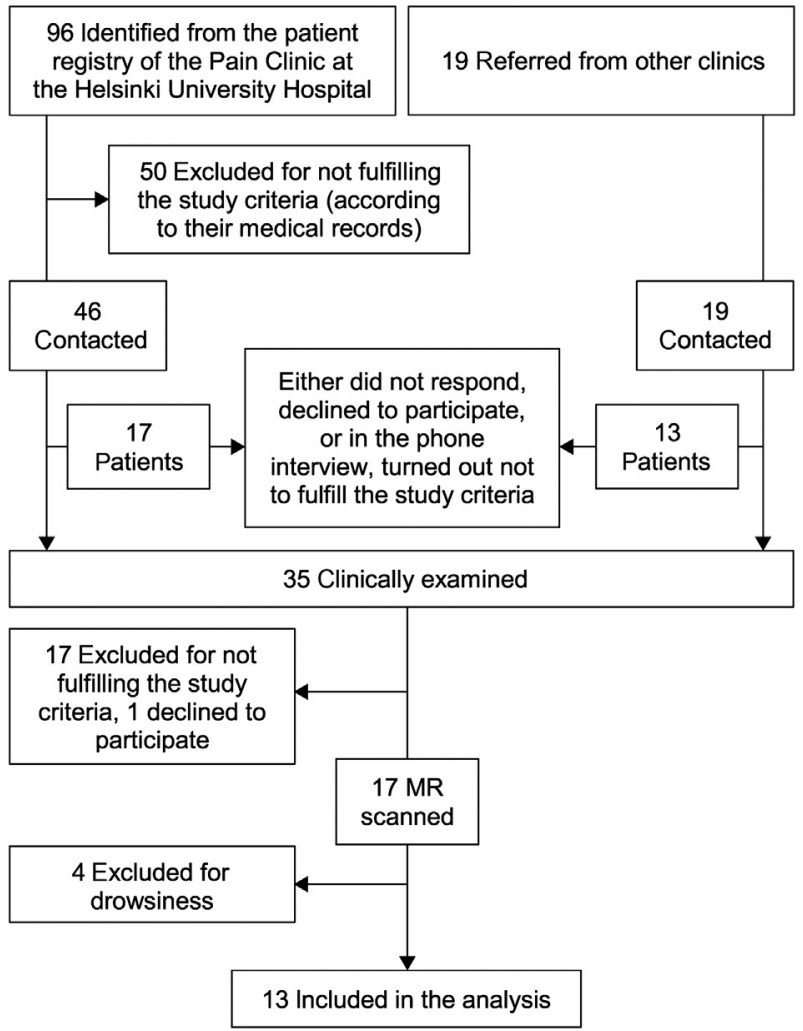

Figure 1. Patient selection. (A) Inclusion and exclusion criteria for the CRPS patients. (B) Patients were recruited primarily from the Pain Clinic at the Helsinki University Hospital where we searched the records from year 2007 to 2013 for patients with a diagnosis of CRPS. In addition, we requested eligible patients from other pain clinics, mainly in Uusimaa district. Of the $115 \mathrm{pa}$ tients initially considered as possible subjects according to their diagnosis, 35 were eligible and were clinically examined by an experienced neurologist with a subspecialty in pain medicine. From this sample, 17 patients fulfilled the study criteria and participated in the fMRI experiment. Eventually, 13 patients presented sufficient alertness during the scan to be included in the analysis.

All the analyzed subjects were female with a mean \pm SD age of $44.7 \pm 6.9$ years (range $=31-58$ years) in the patient group and $44.1 \pm 8.6$ years (range $=29$ 58 years) in the control group. Three patients had a diagnosis of migraine but otherwise none of the subjects had diagnoses of chronic neurological or psychiatric diseases. The control subjects reported no ongoing or long-lasting pain. 
Table 1. Patients Demographic Characteristics and Clinical Data

\begin{tabular}{|c|c|c|c|c|c|c|c|c|c|c|}
\hline \multirow[b]{2}{*}{ CODE } & \multirow[b]{2}{*}{$\begin{array}{l}\text { AGE, } \\
\text { YEARS }\end{array}$} & \multirow[b]{2}{*}{$\begin{array}{c}\text { AFFECTED } \\
\text { HAND }\end{array}$} & \multirow{2}{*}{$\begin{array}{c}\text { SYMPTOM } \\
\text { DURATION, } \\
\text { YEARS }\end{array}$} & \multicolumn{2}{|c|}{$\begin{array}{c}\text { MAXIMUM } \\
\text { OF PAIN }(N R S-11) *\end{array}$} & \multicolumn{4}{|c|}{ SIGNS/SYMPTOMS } & \multirow[b]{2}{*}{ MEDICATION $\dagger$} \\
\hline & & & & REST & MOVEMENT & SENSORY & VASOMOTOR & $\begin{array}{l}\text { SUDOMOTOR } \\
\text { OR EDEMA }\end{array}$ & $\begin{array}{c}\text { MOTOR } \\
\text { OR TROPHIC }\end{array}$ & \\
\hline P01 & 31.0 & Left & 3.5 & 9 & 9 & 以/L & 以/L & 以/L & 以/ & 1,3 \\
\hline P02 & 35.9 & Right & 3.3 & 8 & 8 & レ/L & VIV & レ/L & VIV & $\begin{array}{l}\text { 3, 5, 7, buprenorphine, } \\
\text { clonazepam\# }\end{array}$ \\
\hline P03 & 38.1 & Right & 1.5 & 7 & 10 & V/V & $x / \nu$ & $x / \nu$ & VIV & $1,3,4,5,8$ \\
\hline P04 & 43.1 & Right & 8.2 & 6 & 7 & V/V & VIV & $x / \nu$ & V/v & $\begin{array}{l}1,2,6 \text {, ebastine, } \\
\text { melatonin }\end{array}$ \\
\hline P05 & 44.1 & Right & 8.3 & 10 & 10 & VIV & VIV & $x / \nu$ & V/v & $1 \#, 2,3,4,6$ \\
\hline P06 & 44.5 & Right & 7.5 & 4 & 8 & 以/L & 以/L & 以/L & $x / \nu$ & 2\#, 4\# \\
\hline P07 & 44.7 & Left & 4.2 & 10 & 9 & 以/L & 以/ & 以/L & 以/レ & 1\#, 3\#, orphenadrine\# \\
\hline P08 & 47.1 & Right & 15.5 & 4 & 5 & W/L & VIV & $x / \nu$ & $x / \nu$ & quetiapine, zolpidem \\
\hline P09 & 47.8 & Right & 3.2 & 6 & 6 & V/V & VIV & $x / \nu$ & V/v & 1\#, 2\#, 4\#, lamotrigine \\
\hline P10 & 48.1 & Right & 2.0 & 9 & 5 & W/L & V/v & $x / \nu$ & W/L & $2 \#, 8 \#$ \\
\hline P11 & 49.5 & Left & 3.5 & 7 & 7 & レ & 以/L & 以/L & 以/L & $1,4,7$ \\
\hline P12 & 50.4 & Right & 2.1 & 6 & 9 & 以/V & 以/L & 以/L & 以/L & $1 \#, 2,3,5,6$ \\
\hline P13 & 57.9 & Right & 5.0 & 7 & 7 & レ/L & $x / \nu$ & $x / x$ & 以/L & $2,5,6$ \\
\hline
\end{tabular}

Abbreviation: NRS-11, 11-point numeric rating scale.

NOTE. Gender for all patients was female. The maximum level of experienced pain during rest and during movement on the previous week were evaluated using the NRS-11 ( $0=$ no pain, $10=$ extreme pain). The signs and symptoms were surveyed and evaluated by an experienced neurologist ( $\boldsymbol{}$ indicates present; $\boldsymbol{X}$, not present). All but 1 of the patients in the current study participated in a study published earlier ${ }^{26}$ in which these data were presented in part.

* During the previous week.

$\dagger 1=$ weak opioid, 2 = nonsteroidal anti-inflammatory drug, $3=$ gabapentinoid, $4=$ paracetamol, $5=$ tricyclic antidepressant, $6=$ proton pump inhibitor, $7=$ serotoninnorepinephrine reuptake inhibitor, $8=$ tizanidine, $\#=$ on-demand.

$\ddagger$ Allodynia, hypo- or hyperesthesia.

The sensory, vasomotor, sudomotor/edema, and motor/trophic diagnostic categories of CRPS $^{29}$ were evaluated by an experienced neurologist with a subspecialty in pain medicine (see Table 1 for detailed information of patients' clinical and demographic data). All patients fulfilled "the clinical criteria for CRPS" and all but 1 patient (P13) also fulfilled the more stringent criteria "for research purposes" as defined in "the Budapest criteria." 29 The CRPS symptoms were unilateral (right-side affected in 10 patients and left side in 3), and had begun $5.2 \pm 3.9$ years ago (range $=1.5-15.5$ years).

The study was approved by the Ethics Committee, Department of Medicine of the Helsinki and Uusimaa Hospital District and conducted according to the Declaration of Helsinki. The subjects signed an informed consent before participation.

\section{Experimental Design}

During fMRI scanning, the subjects viewed the same 3.2-second video clips of hand actions that we applied in our previous study in which the stimuli appeared unpleasant and even painful for the patients to observe. ${ }^{31}$ In the current experiment, the video clips were shown in 4-clip blocks (Fig 2). Each of the 48 blocks contained videos of hand actions in 1 of 3 possible categories: either left or right hand remaining static (STATIC condition), repeating gentle movements of opening and closing the fist (FIST), or squeezing an object with maximum force (SQUEEZE; the squeezed objects are presented in Supplementary Fig 1). The subjects were instructed to view the videos without imagining the observed hand movements; their performance on this task was not controlled. In addition, it was emphasized that the hands should be kept still during the stimuli. After the scanning, the subjects evaluated their alertness in the beginning, middle, and end of the scanning session on an 11-point numeric rating scale from 0 (very tired) to 10 (very alert).

To familiarize the subjects with the hand actions, before scanning we asked them to perform the actions and then view the video stimuli (for the protocol details, see Hotta et $\mathrm{al}^{31}$ ). The stimuli were shown in conditionwise blocks, and after each block, the subjects rated their level of pain and valence during the observation on the 11-point numeric rating scale (pain: $0=$ no pain, $10=$ extreme pain; valence: $0=$ very pleasant, $5=$ neutral, $10=$ very unpleasant). Fig 3 shows these ratings (mean \pm standard error of the mean) and illustrates how the unpleasantness increased in order of observing STATIC, FIST, and SQUEEZE stimuli for the patients; all these patients' data (except for 1 patient) were included in our earlier publication comprising altogether 19 CRPS type 1 patients and 19 healthy control subjects. ${ }^{31}$

\section{fMRI Data Acquisition}

The fMRI data were acquired at the Advanced Magnetic Imaging Centre of Aalto Neurolmaging, Aalto University School of Science, 2 to 27 days (mean $13 \pm 6$ SD days) after the recruitment, using 2 MR scanners (because of scanner change in the middle of the study). Nine patients and 9 control subjects were scanned with a Signa 


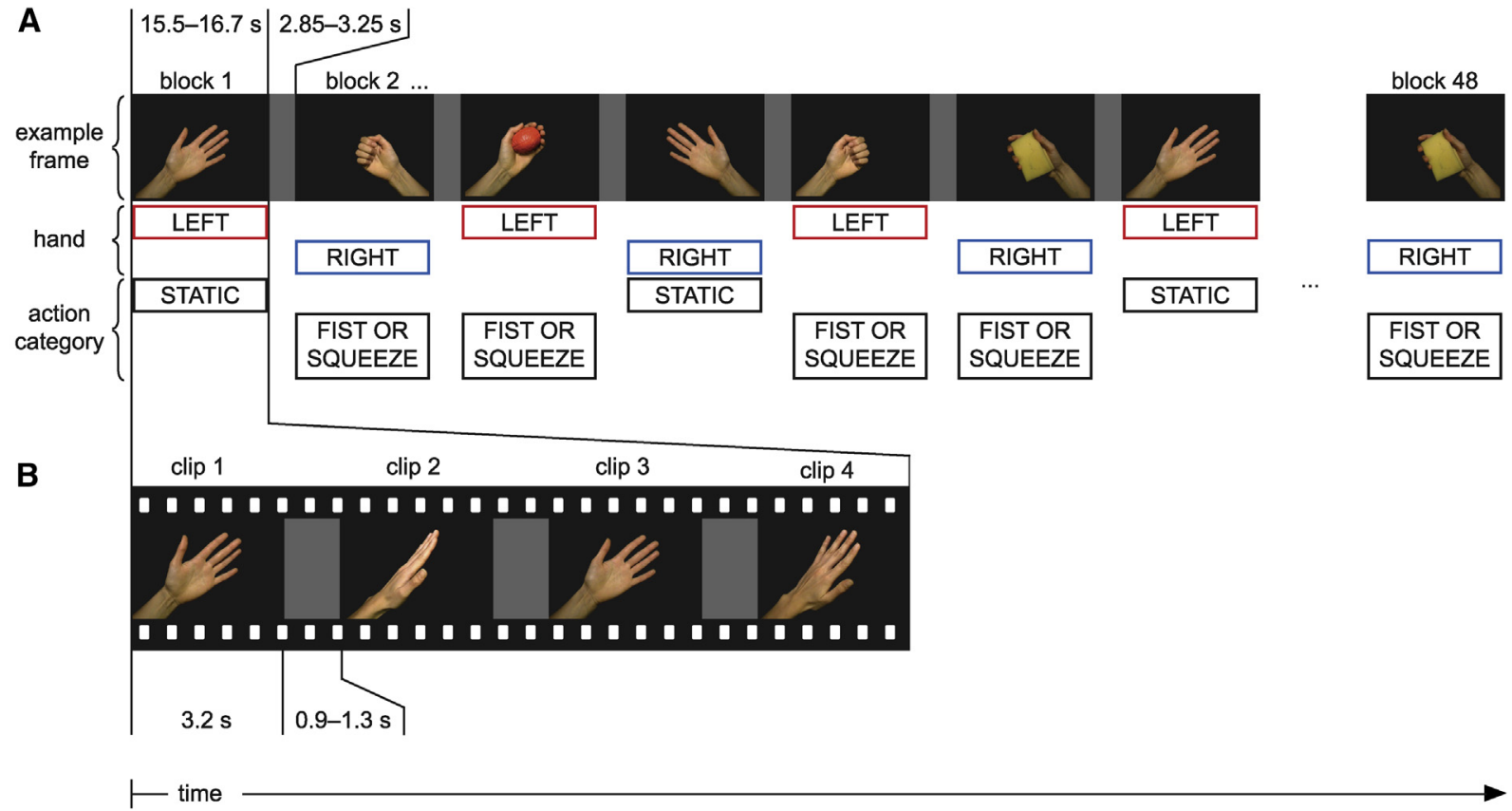

Figure 2. The stimulus protocol and the block structure. (A) The stimuli comprised 48 blocks of hand-action videos presented in a semirandomized order. Each block had 4 video clips of the same female hand performing movements in 1 of the 3 action categories (STATIC, FIST, or SQUEEZE). Four different presentation orders for the blocks were randomized across the subjects. Blocks for left and right hand always alternated. Every third block was always of the STATIC category, and in between, FIST and SQUEEZE blocks were presented randomly. Blocks lasted for 15.5 to 16.7 seconds, with a 2.85- to 3.25-second interblock interval. (B) Each block comprised 4 different 3.2-second video clips of female hand actions, with .9- to 1.3-second interclip intervals. Altogether, 8 such clips were included for each action category and each clip presented the hand from a slightly different angle in the first-person view. Each clip was presented 4 times during the experiment. The order of the video clips within a block was randomized.

HDxt 3T scanner (GE Healthcare [GE], Milwaukee, WI) with a 16-channel head coil, and 4 patients and 4 control subjects with a Magnetom Skyra 3T scanner (Siemens Healthcare [Siemens], Erlangen, Germany) with a 30-channel head coil (modified from the standard 32-channel head coil to optimize visual field of view). Presentation software (NeuroBehavioral Systems, Berkeley, CA) and a Vista X3 projector (Christie Digital, Cypress, $\mathrm{CA}$ ) were used to deliver the videos to the subjects via a mirror system.

During stimulus presentation, functional $\mathrm{T}_{2}$ * $_{-}$ weighted gradient-echo echo-planar images were acquired with the following parameters: repetition time 2.5 seconds, echo time $30 \mathrm{~ms}$, flip angle $75^{\circ}$, matrix size $64 \times 64$, field of view $24 \mathrm{~cm}$, slice thickness $3.0 \mathrm{~mm}$ with no gap, in-plane resolution $3.75 \times 3.75 \mathrm{~mm}^{2}$, and the number of slices 50 (GE) or 47 (Siemens). The number of time points was 410 , resulting in a total scan time of 17 minutes and 30 seconds. The first 8 volumes were discarded to ensure MR signal stabilization. During the same scanning session, anatomical high-resolution $1 \times 1 \times 1 \mathrm{~mm}^{3} \mathrm{~T} 1$ weighted MR images with 176 slices of matrix size $256 \times 256$ were acquired using ultrafast gradientecho 3-D sequences (3-D fast spoiled gradient-recalled sequence with GE scanner, magnetization-prepared rapid-acquisition sequence with Siemens scanner) with repetition time $10.0 \mathrm{~ms} / 2,530 \mathrm{~ms}$, echo time $2.9 \mathrm{~ms} / 3.3 \mathrm{~ms}$, flip angle $15^{\circ} / 7^{\circ}$ for GE/Siemens, respectively.

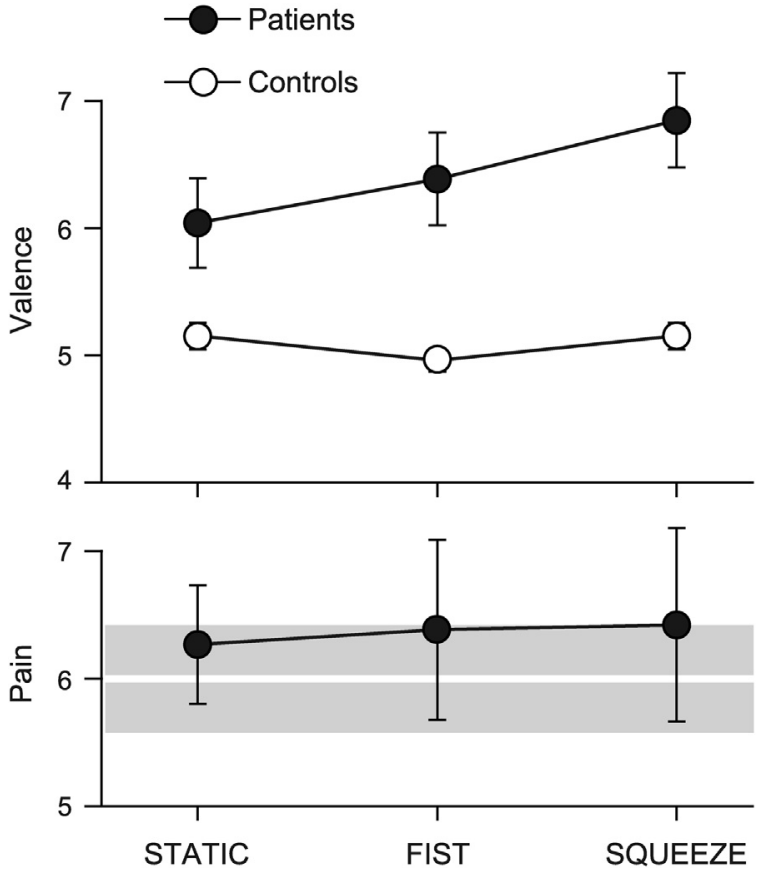

Figure 3. Valence and pain ratings for the stimuli. The mean \pm standard error of the mean valence (upper panel) and pain (lower panel) ratings presented separately for STATIC, FIST, and SQUEEZE stimuli. The white line represents the mean pre-experiment pain level in the patients (gray area = standard error of the mean). The healthy control subjects did not report any pain during the stimuli; for illustrative purposes only their valence ratings are presented. All these data, except for $1 \mathrm{pa}$ tient, are a part of a larger data sample presented in our earlier publication. $^{31}$ 
Drowsiness was monitored by following eye closures with an eye-tracker (iView X MRI-LR, SensoMotoric Instruments $\mathrm{GmbH}$, Germany, in the GE scanner; EyeLink 1000, SR Research Ltd, Ontario, Canada, in the Siemens scanner). Moreover, the subjects' hand movements were monitored with 2 custom-made MR-compatible accelerometers attached to fingers 3 through 5 of both hands (data collected using BrainAmp ExG MR amplifiers; Brain Products $\mathrm{GmbH}$, Munich, Germany).

\section{fMRI Preprocessing and General Linear Model}

The fMRI data were preprocessed and normalized to the anatomical Montreal Neurological Institute space using SPM8 software (http://www.fil.ion.ucl.ac.uk). The fMRI preprocessing included slice-time correction, motion correction, smoothing (6-mm full-width at halfmaximum isotropic Gaussian kernel), coregistration of the skull-stripped functional images (using the mean image) with corresponding T1 images, and normalization of data to the Montreal Neurological Institute anatomical space using the Colin template (http://wwww.bic. mni.mcgill.ca/ServicesAtlases/Colin27). The normalized voxel size was $3.75 \times 3.75 \times 3 \mathrm{~mm}^{3}$. Finally, a high-pass filter (cutoff at $.01 \mathrm{~Hz}$ ) was applied to remove lowfrequency signal drifts.

The general linear model (GLM) was used to estimate the level of brain activation ( $\beta$-values) for each of the 6 observation conditions (STATIC, FIST, and SQUEEZE, separately for left and right hands). In the GLM, the time courses of the 6 conditions were modeled as boxcar functions convolved with the canonical hemodynamic response function. To reduce movement-related effects in the $6 \beta$-values, the estimated movement parameters ( 3 translations and 3 rotations) were included as nuisance regressors in the GLM. Multivariate group analyses were limited to gray matter using the corresponding SPM8 mask (http://www.fil.ion.ucl.ac.uk), resulting in 32,868 analyzed voxels.

\section{Multivariate Analysis}

We trained a set of classifiers to find brain activity patterns that would discriminate CRPS patients from the control group. We used the searchlight method ${ }^{36}$ that bases classification of $\mathrm{fMRI}$ data on activity patterns in spherical volumes; in this study we used volumes of 123 adjacent voxels, corresponding to a sphere of 7 voxels (approximately $2.1 \mathrm{~cm}$ ) in diameter, if not cropped smaller by the gray matter mask. In the searchlight procedure, each gray matter voxel acts once as a center voxel of such sphere (ie, "searchlight"), resulting in spatial maps of classification accuracies assigned to the center voxels. In other words, the multivariate pattern analysis with the searchlight method provides information about where in the brain and with what probability (accuracy) the local brain responses differ between subject groups.

\section{Statistical Analysis}

GLM $\beta$-value contrasts (Table 2), 1 for each voxel, were used as classification features. The classification
Table 2. Contrasts Applied in fMRI Analysis

\begin{tabular}{|c|c|c|c|c|c|c|}
\hline & \multicolumn{2}{|c|}{ Static } & \multicolumn{2}{|c|}{ FIST } & \multicolumn{2}{|c|}{ SQUEEZE } \\
\hline & RIGHT & LEFT & RIGHT & LEFT & RIGHT & LEFT \\
\hline STATIC & + & + & & & & \\
\hline FIST & & & + & + & & \\
\hline SQUEEZE & & & & & + & + \\
\hline SQUEEZE - STATIC & - & - & & & + & + \\
\hline SQUEEZE - FIST & & & - & - & + & + \\
\hline FIST - STATIC & - & - & + & + & & \\
\hline PAIN - NO-PAIN* & + & - & + & - & + & - \\
\hline
\end{tabular}

NOTE. For the group analyses, 7 different $\beta$-value contrasts were calculated individually. In the table, the first column presents the contrast name. Columns 2 to 7 indicate the stimuli for which $\beta$-values were summed $(+)$ or subtracted $(-)$ for each contrast. First, to study the effects of each action category (regardless of the presented hand), the category-specific sum of $\beta$-values for left and right hand stimuli were computed for each of 1) STATIC, 2) FIST, and 3) SQUEEZE categories. These plain contrasts are represented in the first 3 rows of the table. Second, to highlight the differences between action categories (regardless of the presented hand), the category-specific sum of $\beta$-values for left and right hand stimuli were subtracted pairwise between categories 4) SQUEEZE - STATIC, 5) SQUEEZE - FIST, and 6) FIST - STATIC contrasts. Third, to study whether patients respond abnormally to observing hands corresponding to their affected hand, the sum of $\beta$-values for stimuli presenting the healthy-sided hand was subtracted from the sum of $\beta$-values for stimuli presenting the hand of the affected side: 7) PAIN - NO-PAIN contrast (in the table, the contrast weights are for patients with affected right side). For each age-matched control subject, corresponding hand-matched calculations were performed.

*For the patients with affected left side (and the corresponding control subjects), contrast weights were of opposite signs.

accuracies were calculated with leave-2-subjects-out cross-validation so that 1 patient and 1 control subject were left out (altogether $13 \times 13=169$ patientcontrol pairs) for testing, with the classifier trained by the data of the remaining 24 subjects. In each voxel, the final classification accuracy was the percentage of correct classifications over all 169 patientcontrol pairs.

We classified 7 different $\beta$-value contrasts separately (STATIC, FIST, SQUEEZE, FIST - STATIC, SQUEEZE - STATIC, SQUEEZE - FIST, and PAIN - NO-PAIN; Table 2). Support vector machine was used with the regularization parameter $C=1^{62}$ as implemented in the PyMVPA toolbox (http://www.pymvpa.org). ${ }^{26}$ The statistical significance of the classification performance was estimated using permutation testing ${ }^{49}$ separately for each searchlight ( $n=32,868$ ): the null-distributions for the classification accuracies were estimated by randomly permuting the labels (patient/control) before the cross-validation procedure 5,000 times.

The $P$ values derived from the estimated null distribution were corrected for multiple comparisons $(n=32,868)$ using false discovery rate ${ }^{24}$ with $q<.05$.

\section{Results}

\section{Classification of Brain Responses}

Statistically significant classification performances varied from 86.4 to $100 \%$, with highest accuracies for SQUEEZE and SQUEEZE - STATIC contrasts.

Fig 4 shows the classification results for the SQUEEZE contrast. The classification accuracy was 
A

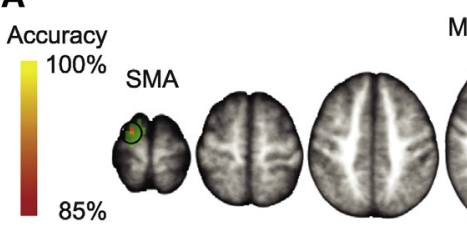

74

62

47

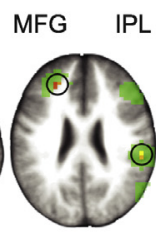

26

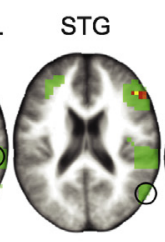

20

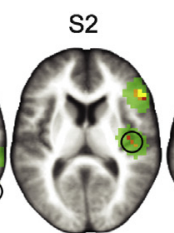

11

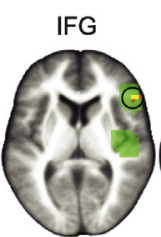

8

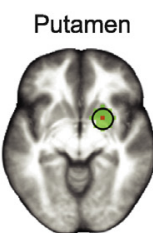

$-6$

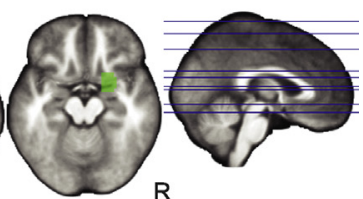

$-11$
B

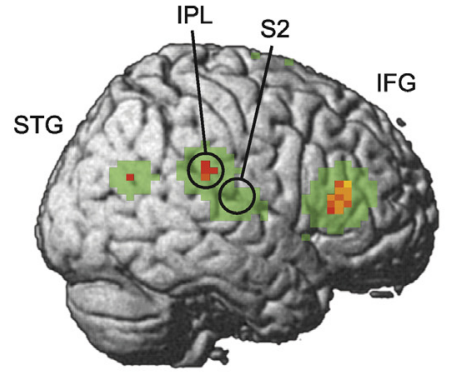

Contrast: SQUEEZE

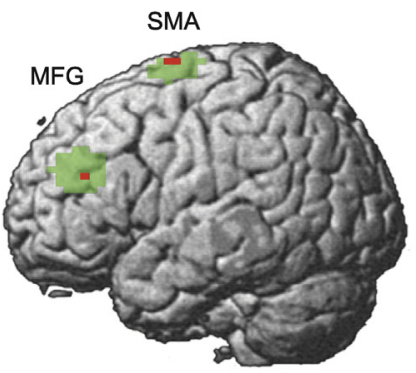

C

논

Decision boundar

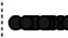

- 00000

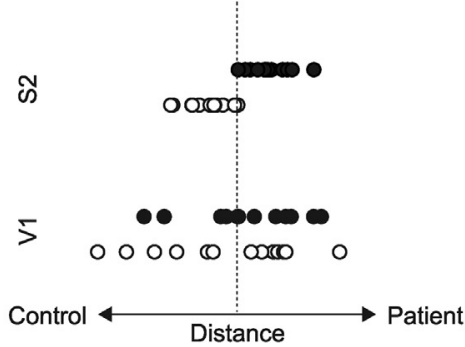

- Patients o Controls

Figure 4. Classification results for the SQUEEZE contrast. (A) Brain areas contributing to statistically significant searchlight classifications are presented in green and their respective center voxels on a red-yellow gradient. The results are overlaid on a mean T1-image of the whole subject group. (B) The corresponding results on a 3-D template brain (SPM8; http://www.fil.ion.ucl.ac.uk). (C) The subjectwise mean distances from the decision boundary presented separately for 3 exemplar searchlight areas: inferior frontal gyrus (IFG; classification accuracy of $100 \%$ ), secondary somatosensory cortex (S2; classification accuracy of $95 \%$ ), and primary visual cortex (V1; classification accuracy of 45\%). During the classification procedure, a decision boundary was created for each training set (12 patients, 12 control subjects) and the remaining subjects (1 patient, 1 control) were considered as patients or control subjects depending on which side of the boundary they fell; in the figure, the subjects were classified as patients if they are plotted on the right side of the boundary, and healthy if they are on the left side. The decision boundary is marked with the dotted vertical line. Abbreviations: MFG, middle frontal gyrus; IPL, inferior parietal lobule; STG, superior temporal gyrus; R, right; SMA, supplementary motor area.

statistically significant $(q<.05$, false discovery ratecorrected) in 7 distinct and lateralized brain regions. The most notable regions were the right pars triangularis (mainly Brodmann area 45) in the inferior frontal gyrus, the right secondary somatosensory cortex, the right supramarginal gyrus in the inferior parietal lobule, and the middle and superior frontal gyri (supplementary motor area) in the left hemisphere (for detailed information about brain regions, cytoarchitectonic areas, coordinates, and classification accuracies, see Table 3 ).

Fig 5 shows the corresponding classification performance for the SQUEEZE - STATIC contrast. Statistically significant classifications were observed in 7 separate regions, the most prominent one in the left sensorimotor hand area (the hand $\mathrm{knob}^{68}$ ). One notable region covered large parts of the right thalamus (extending also to the caudate), whereas another region was located in the right middle orbitofrontal gyrus (for detailed information, see Table 3).

For the other plain contrasts (STATIC, FIST), as well as for the other contrasts addressing differences between conditions (SQUEEZE - FIST, FIST - STATIC) or between observed hands (PAIN - NO-PAIN), statistically significant classifications were much less frequent (by a factor of 3-10 compared with SQUEEZE or SQUEEZE - STATIC contrasts), but the accuracies were still high, ranging from 86 to $98 \%$ (for details, see Supplementary Table 1).

\section{Patients With Right-Sided Symptoms}

Because some brain changes in chronic pain patients depend on the affected side (see Vartiainen et $\mathrm{al}^{6}{ }^{63}$ for example), the searchlight classifications were also performed with a subgroup of patients with right-hand pain $(n=10)$ and their optimally matched control subjects $(n=10)$.

In this subject sample, the searchlight analysis produced statistically significant classifications mainly for the SQUEEZE - STATIC contrast (accuracies from 91$100 \%)$. Most of these successful classifications were in the same regions as for the original groups of 13 subjects, that is, in the left sensorimotor hand area and the right middle orbitofrontal gyrus. As for the other contrasts, the most notable classification accuracies appeared for the PAIN - NO-PAIN contrast in the supplementary motor area and adjacent middle cingulate gyrus. For details, see Supplementary Table 2 and Supplementary Fig 2.

\section{Confounding Factors}

To rule out spurious findings, we also performed patient versus control classification using features from head movements, hand movements, and measures of alertness (for details, see the Supplementary File). This analysis resulted in a statistically significant classification accuracy of $70 \%(P=.045$; lower bound of statistical significance $=69.5 \%$ ). However, separate univariate 
Table 3. Classification Results for SQUEEZE and SQUEEZE - STATIC Contrasts

\begin{tabular}{|c|c|c|c|c|c|c|c|c|c|}
\hline \multirow[b]{2}{*}{ CONTRAST } & \multirow[b]{2}{*}{ CORTEX } & \multirow[b]{2}{*}{ REGION } & \multirow{2}{*}{$\begin{array}{l}\text { CYTOARCHITECTONIC } \\
\text { AREA }\left(\geq 1 \mathrm{CM}^{3}\right)\end{array}$} & \multicolumn{2}{|c|}{ Cluster Size } & \multirow{2}{*}{$\begin{array}{c}\text { ACCURACY, \% } \\
\text { RANGE }\end{array}$} & \multicolumn{3}{|c|}{ COORDINATE* } \\
\hline & & & & SEARCHLIGHTS & VOXELS & & $x$ & $Y$ & $z$ \\
\hline \multirow[t]{7}{*}{ SQUEEZE } & Frontal & $\begin{array}{l}\text { R inferior frontal gyrus } \\
\text { (pars triangularis) }\end{array}$ & Area $45, \mathrm{BA} 48 \dagger$ & 31 & 335 & $89.1-100$ & 53 & 29 & 11 \\
\hline & & L middle frontal gyrus & BA $46 \dagger$ & 4 & 135 & 89.9-94.7 & -23 & 40 & 26 \\
\hline & & $\begin{array}{l}\text { L superior frontal gyrus } \\
\text { (SMA) }\end{array}$ & BA $6 \dagger$ & 2 & 77 & $92.0-93.8$ & -19 & -5 & 74 \\
\hline & Parietal & R supramarginal gyrus & $\begin{array}{l}\text { PFcm, PF, PFop, } \\
\text { OP1, BA 48† }\end{array}$ & 5 & 220 & $91.7-99.1$ & 56 & -28 & 26 \\
\hline & Temporal & $\begin{array}{l}\text { R secondary somatosensory } \\
\text { cortex }\end{array}$ & $\begin{array}{l}\text { OP3, OP1, } \\
\text { BA 48† }\end{array}$ & 3 & 209 & $91.1-95.3$ & 49 & -20 & 11 \\
\hline & & R superior temporal gyrus & PGp & 1 & 61 & 90.8 & 60 & -65 & 23 \\
\hline & Subcortical & R putamen & BA $48+$ & 1 & 116 & 91.7 & 23 & 6 & -7 \\
\hline \multirow[t]{9}{*}{ SQUEEZE - STATIC } & Frontal & $\mathrm{R}$ middle orbital gyrus & Fp1, BA 47† & 7 & 129 & $88.5-99.4$ & 34 & 59 & -16 \\
\hline & & $R$ and $L$ paracentral lobule & Area 4, BA 4† & 1 & 122 & 89.4 & 0 & -24 & 62 \\
\hline & Parietal & L postcentral gyrus & $\begin{array}{l}\text { Area } 4,2,3, \\
\text { BA } 4 \dagger, \text { BA } 6 \dagger\end{array}$ & 16 & 276 & $87.3-92.9$ & -38 & -31 & 59 \\
\hline & & L postcentral gyrus & $\mathrm{BA} 3 \dagger$ & 1 & 73 & 91.1 & -45 & -31 & 62 \\
\hline & & L postcentral gyrus & Area 2 & 1 & 61 & 89.3 & -49 & -39 & 59 \\
\hline & & $\mathrm{R}$ rolandic operculum & OP1, PFop & 1 & 121 & 91.7 & 56 & -20 & 20 \\
\hline & Occipital & $\begin{array}{l}\mathrm{R} \text { and } \mathrm{L} \text { calcarine and } \\
\text { lingual gyrus }\end{array}$ & Area 17 & 1 & 98 & 89.6 & 8 & -95 & -4 \\
\hline & Insular & $\begin{array}{l}\mathrm{L} \text { insula and parts of } \\
\text { putamen }\end{array}$ & BA $48 \dagger$ & 1 & 109 & 92.9 & -30 & 10 & 8 \\
\hline & Subcortical & $\begin{array}{l}\mathrm{R} \text { thalamus, extending to } \\
\text { caudate }\end{array}$ & $\begin{array}{l}\text { Th-prefrontal, } \\
\text { Th-temporal }\end{array}$ & 6 & 174 & $91.1-95.9$ & 15 & -13 & 11 \\
\hline
\end{tabular}

Abbreviations: BA, Brodmann area; R, right; L, left; SMA, supplementary motor area; OP, operculum; Fp1, frontopolar area 1; Th, thalamus; MNI, Montreal Neurological Institute; S2, secondary somatosensory cortex.

NOTE. Classification results for SQUEEZE and SQUEEZE - STATIC contrasts. Each row presents the localization (columns 2-4), the size (columns 5-6), the range of classification accuracies (column 7), and the MNI coordinate of the highest accuracy (last 3 columns) of 1 cluster of adjacent center voxels of searchlights with statistically significant classifications. The cytoarchitectonic areas (column 4) are primarily derived from the SPM extension Anatomy Toolbox v2.0. ${ }^{18-20}$ Areas unassigned by the Anatomy Toolbox are labeled using the Brodmann template. ${ }^{60}$ The cluster size is presented in the number of adjacent searchlights and, in addition, in the number of voxels these searchlights cover. Anatomy Toolbox areas: area 2 and 3 in the primary somatosensory cortex; area 4 in the motor cortex; area 17 in the occipital cortex; area 45 in Broca region; Fp1 in the frontal pole; OP1 and OP3 in the parietal operculum and S2; PF, PFcm, PFop, and PGp in the inferior parietal cortex; Th-prefrontal and Th-temporal in the thalamus.

*Center coordinate (MNI) of the searchlight with highest classification accuracy.

†MRIcron: Brodmann template.

Wilcoxon tests $(P<.05$, Bonferroni-corrected) did not show factorwise differences between the groups.

\section{Discussion}

In $\mathrm{fMRI}$ recordings performed during action observation, CRPS patients displayed abnormal brain responses. The abnormalities were most robust for videos showing object manipulation with high force (SQUEEZE) that, according to our previous study, ${ }^{31}$ is perceived as unpleasant or even painful in CRPS patients. For these videos, the pattern classification revealed group differences in parts of the action observation network (inferior frontal gyrus and inferior parietal lobule; for a review, see Caspers et $\mathrm{al}^{10}$ ), and in areas related to pain perception (secondary somatosensory cortex) or motor control (supplementary motor area). Also, the contrast between the brain responses to the most unpleasant and to the least unpleasant stimuli (SQUEEZE - STATIC) revealed notable group differences. These differences appeared mainly in pain-responsive brain areas (primary sensorimotor cortex and thalamus) and in the orbitofrontal cortex, an area likely participating in pain modulation. ${ }^{52,66}$
With the SQUEEZE contrast, the most prominent group differences were in the right inferior frontal gyrus, an area included in a frontal network that mediates effects of attention, expectations, and reappraisal during pain perception. ${ }^{67}$ Lamm et $\mathrm{al}^{37}$ proposed, in an fMRI study addressing empathy for pain in healthy subjects, that the right inferior frontal gyrus inhibits aversive responses during observation of painful situations. More generally, the right inferior frontal gyrus is known to participate in motor and cognitive inhibition (for details, see a recent meta-analysis ${ }^{39}$ ). Subjects in both groups kept their hands still as instructed (see Supplementary File), and such immobility during action observation requires suppression of automatic imitation. ${ }^{12,30}$ Taken together, our findings in the right inferior frontal gyrus might reflect group differences in the salience and quality of inhibited responses to action observation. Because of unpleasantness of SQUEEZE stimuli for the patients, a dysfunctional inhibition related to aversive associations with motor actions might also explain the activation differences. Such dysfunction could be an important pathophysiological mechanism explaining many central nervous system-related features of CRPS. 
A

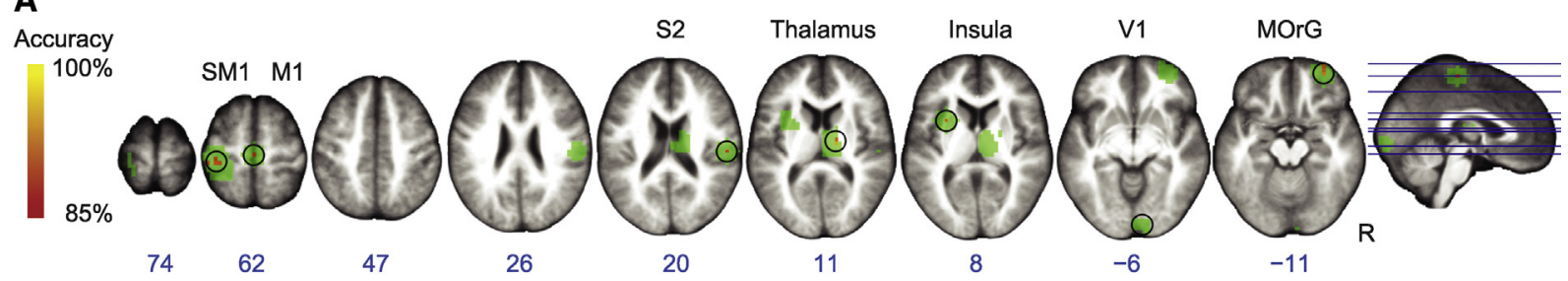

B

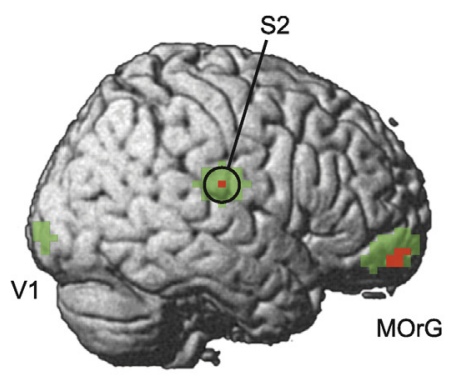

Contrast: SQUEEZE - STATIC

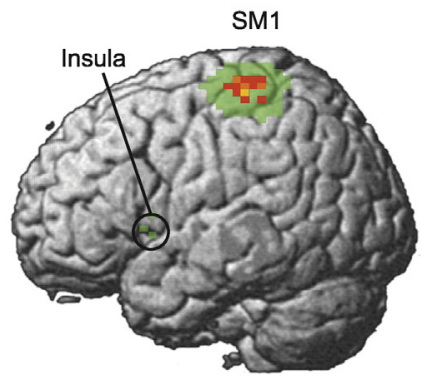

C

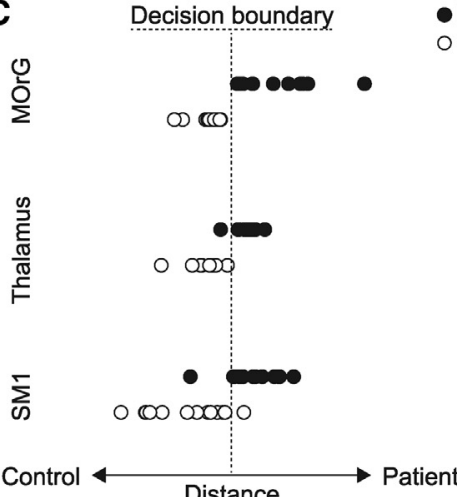

Figure 5. Classification results for the SQUEEZE - STATIC contrast. Markings similar as in Fig 4 for panels (A) and (B). (C) The subjectwise mean distances from the decision boundary presented separately for 3 exemplar searchlight areas: middle orbital gyrus (MOrG; classification accuracy of $99 \%$ ), thalamus (classification accuracy of $96 \%$ ), and primary sensorimotor cortex (SM1; classification accuracy of $93 \%$ ). During the classification procedure, a decision boundary was created for each training set (12 patients, 12 control subjects) and the remaining subjects (1 patient, 1 control) were considered as patients or control subjects depending on which side of the boundary they fell; in the figure, the subjects were classified as patients if they are plotted on the right side of the boundary, and healthy if they are on the left side. The decision boundary is marked with the dotted vertical line. Abbreviations: M1, primary motor cortex; S2, secondary somatosensory cortex; V1, primary visual cortex; R, right.

Of special importance are the group differences in the left primary sensorimotor cortex-especially in the subanalysis of the right limb patients-because they coincide with the hand representation area (SQUEEZE - STATIC contrast). During action observation, the activity of the primary somatosensory cortex is modulated somatotopically in relation to the observed body part, ${ }^{1,48,58}$ and likely accounts for simulation of the somatosensory content of the observed action (for a review, see Keysers et $\mathrm{al}^{33}$ ). The somatosensory cortex is known to display abnormal representations of the painful hand in CRPS patients, ${ }^{17,32}$ associated with disinhibition in the adjacent motor cortex. 21,32,34,56 Our finding indicates that the dysfunction in the sensorimotor cortex also affects the neural processing of observed actions.

Many of the brain areas that showed successful classifications in our study are known to show abnormal activation in CRPS patients during action execution, and they have been associated with hyperalgesia and allodynia in CRPS, ${ }^{40-42}$ which increases the functional reliability of our findings.

Action execution and observation are associated with similar changes in neuronal activity in many brain areas. Abnormal brain activations during action observation can thus be informative of neural processes underlying motor dysfunction. Also, action observation has been studied as a motor rehabilitation therapy to normalize motor circuitry after ischemic stroke and in Parkinson dis- ease (for a recent review, see Buccino ${ }^{7}$ ). In patients with CRPS, action observation also could be used as a gentle way to start rehabilitation, for example, before graded motor imagery (GMI) therapy. ${ }^{45}$ In theory, action observation could complement GMI because: 1) it might be superior to motor imagery in protecting normal functions of the primary sensorimotor cortex during limb disuse, ${ }^{5}$ and 2) in contrast to motor imagery, ${ }^{25}$ there is now evidence that action observation addresses the disruption in this brain area in CRPS. In GMI, the central motor circuitry is also trained with modulated visual feedback of the patients' own motor actions (mirror-box therapy). Whereas our study addressed visual processing during motor inactivity, future studies should specify the effects of visual feedback on neural processes during motor actions. In conclusion, our current findings of abnormal brain activation during action observation in CRPS are in line with the potential of action observation therapy in CRPS patients.

Recently, classification approaches have become popular in searching for neural signatures of central nervous system diseases, ${ }^{35}$ and, in parallel, chronic pain has gained increasing attention. 2,3,9,59,61 On this basis, our study introduces some considerable novelties. First, we used video stimuli in contrast to static pictures or other conventional visual stimuli. Second, we assessed the discriminatory power of local fMRI patterns across the cortex in contrast to studies using the whole-brain data or preselected brain regions. Third, we used functional 
data instead of structural MR imaging (eg, Bagarinao et al, $^{2}$ Baliki et al, ${ }^{3}$ and Tzourio-Mazoyer et $\mathrm{al}^{61}$ ) where changes plausibly become observable long after functional changes. Fourth, we stimulated the brains via visual pathways that are not, as far as we know, altered in CRPS. The stimulation was thus more similar across all subjects-patients and healthy controls-than what would have been possible to obtain with for example, somatosensory stimuli in patients who suffer from various types of sensorimotor deficits. Additionally, our stimuli were known beforehand to elicit different experiences in the studied subject groups. ${ }^{31}$

However, the purpose of our classification was not to classify individuals in a diagnostic manner but to provide a multivariate approach to find brain areas where the functionality differs between 2 subject groups. Although our results can be beneficial for the development of a biomarker for CRPS, the requirements for clinical brain imaging biomarkers ${ }^{13}$ were out of the scope of the study. For biomarker development, considerably larger group sizes would be needed, the findings should be validated with a separate cohort with more variable patient characteristics, and the specificity for CRPS should be determined with the inclusion of patients suffering from other types of chronic pain or movement disorders. The potential for a biomarker is, however, supported by the findings that the data collected with 2 different MR scanners showed similar discriminative response characteristics between the subject groups.

We note that our sample size is relatively small, which decreases the positive predictive value of our results (see, for example, Button et $\mathrm{al}^{8}$ ) and increases the likelihood for perfect classification accuracies (up to 100\%). ${ }^{11}$ Thus, our results should be considered tentative until confirmed with an independent and larger sample. Moreover, although data confounds (head or hand movements, or variation of alertness) did not differ between groups in factorwise univariate analyses, they discriminated the subject groups in multivariate analysis

\section{References}

1. Avikainen S, Forss N, Hari R: Modulated activation of the human SI and SII cortices during observation of hand actions. Neuroimage 15:640-646, 2002

2. Bagarinao $E$, Johnson KA, Martucci $K T$, Ichesco $E$, Farmer MA, Labus J, Ness TJ, Harris R, Deutsch G, Apkarian AV, Mayer EA, Clauw DJ, Mackey S: Preliminary structural MRI based brain classification of chronic pelvic pain: A MAPP Network Study. Pain 155: 2502-2509, 2014

3. Baliki MN, Schnitzer TJ, Bauer WR, Apkarian AV: Brain morphological signatures for chronic pain. PLoS One 6: e26010, 2011

4. Bass C: Complex regional pain syndrome medicalises limb pain. BMJ 348:g2631, 2014

5. Bassolino M, Campanella M, Bove M, Pozzo T, Fadiga L: Training the motor cortex by observing the actions of others during immobilization. Cereb Cortex 24: 3268-3276, 2014 with an accuracy of $70 \%$, suggesting that our data might incorporate some confounding effects.

We also note that the patients were receiving medication, which is a common confound in any study of chronic diseases; for example, $69 \%$ of our patients used weak opioids or buprenorphine (one patient). Opioids are known to suppress pain responses in primary and secondary somatosensory cortex and insula ${ }^{51}$ and decrease the classification accuracies between painful and nonpainful stimuli in the primary somatosensory cortex. ${ }^{65}$ Thus, in contrast to increasing group differences, the medication might hamper them and affect the classification accuracies negatively.

\section{Conclusions}

Our approach to use video stimuli, unpleasant for patients and neutral for healthy subjects, proved successful in producing discriminative fMRI signatures for CRPS. These signatures appeared in functionally feasible brain areas, which increases the reliability of our results and confirms that, in CRPS, the central motor and sensory circuitries are compromised to a level that also affects processing of action observation.

\section{Acknowledgments}

We acknowledge Prof. Eija Kalso and Dr. Hanna Harno for patient recruitment, Prof. Lauri Nummenmaa for help in study design, and Radiographer Marita Kattelus for help in MR data collection. We acknowledge the computational resources provided by the Aalto ScienceIT project.

\section{Supplementary Data}

Supplementary data related to this article can be found online at http://dx.doi.org/10.1016/j.jpain.2016. 10.017 .

6. Birklein F, Rowbotham MC: Does pain change the brain? Neurology 65:666-667, 2005

7. Buccino G: Action observation treatment: A novel tool in neurorehabilitation. Philos Trans R Soc Lond B Biol Sci 369: 20130185, 2014

8. Button KS, loannidis JP, Mokrysz C, Nosek BA, Flint J, Robinson ES, Munafò MR: Power failure: Why small sample size undermines the reliability of neuroscience. Nat Rev Neurosci 14:365-376, 2013

9. Callan D, Mills L, Nott C, England R, England S: A tool for classifying individuals with chronic back pain: Using multivariate pattern analysis with functional magnetic resonance imaging data. PLoS One 9:e98007, 2014

10. Caspers S, Zilles K, Laird AR, Eickhoff SB: ALE metaanalysis of action observation and imitation in the human brain. Neuroimage 50:1148-1167, 2010

11. Combrisson E, Jerbi K: Exceeding chance level by chance: The caveat of theoretical chance levels in brain signal classification and statistical assessment of 
decoding accuracy. J Neurosci Methods 250:126-136, 2015

12. Cross KA, Torrisi S, Reynolds Losin EA, lacoboni M: Controlling automatic imitative tendencies: Interactions between mirror neuron and cognitive control systems. Neuroimage 83:493-504, 2013

13. Davis KD, Racine E, Collett B: Neuroethical issues related to the use of brain imaging: Can we and should we use brain imaging as a biomarker to diagnose chronic pain? Pain 153: 1555-1559, 2012

14. de Asla RJ: Complex regional pain syndrome type I: Disease or illness construction? Commentary on an article by Marlies I. Bodde, MD, et al.: "Therapy-resistant complex regional pain syndrome type I: to amputate or not?". J Bone Joint Surg Am 93:e116, 2011

15. de Boer RD, Marinus J, van Hilten JJ, Huygen FJ, van Eijs $F$, van Kleef $M$, Bauer $M C$, van Gestel $M$, Zuurmond WW, Perez RS: Distribution of signs and symptoms of complex regional pain syndrome type I in patients meeting the diagnostic criteria of the International Association for the Study of Pain. Eur J Pain 15:830.e1-830.e8, 2011

16. de Mos M, de Bruijn AG, Huygen FJ, Dieleman JP, Stricker BH, Sturkenboom MC: The incidence of complex regional pain syndrome: A population-based study. Pain 129:12-20, 2007

17. Di Pietro F, McAuley JH, Parkitny L, Lotze M, Wand BM, Moseley GL, Stanton TR: Primary somatosensory cortex function in complex regional pain syndrome: A systematic review and meta-analysis. J Pain 14:1001-1018, 2013

18. Eickhoff SB, Heim S, Zilles K, Amunts K: Testing anatomically specified hypotheses in functional imaging using cytoarchitectonic maps. Neuroimage 32:570-582, 2006

19. Eickhoff SB, Paus T, Caspers S, Grosbras MH, Evans AC, Zilles K, Amunts K: Assignment of functional activations to probabilistic cytoarchitectonic areas revisited. Neuroimage 36:511-521, 2007

20. Eickhoff SB, Stephan KE, Mohlberg H, Grefkes C, Fink GR, Amunts K, Zilles K: A new SPM toolbox for combining probabilistic cytoarchitectonic maps and functional imaging data. Neuroimage 25:1325-1335, 2005

21. Eisenberg E, Chistyakov AV, Yudashkin M, Kaplan B, Hafner H, Feinsod M: Evidence for cortical hyperexcitability of the affected limb representation area in CRPS: A psychophysical and transcranial magnetic stimulation study. Pain 113:99-105, 2005

22. Galer BS, Bruehl S, Harden RN: IASP diagnostic criteria for complex regional pain syndrome: A preliminary empirical validation study. International Association for the Study of Pain. Clin J Pain 14:48-54, 1998

23. Galer BS, Butler S, Jensen MP: Case reports and hypothesis: A neglect-like syndrome may be responsible for the motor disturbance in reflex sympathetic dystrophy (complex regional pain syndrome-1). J Pain Symptom Manage 10: 385-391, 1995

24. Genovese CR, Lazar NA, Nichols T: Thresholding of statistical maps in functional neuroimaging using the false discovery rate. Neuroimage 15:870-878, 2002

25. Gieteling EW, Van Rijn MA, De Jong BM, Hoogduin JM, Renken R, Van Hilten JJ, Leenders KL: Cerebral activation during motor imagery in complex regional pain syndrome type 1 with dystonia. Pain 134:302-309, 2008
26. Hanke M, Halchenko YO, Sederberg PB, Hanson SJ, Haxby JV, Pollmann S: PyMVPA: A python toolbox for multivariate pattern analysis of $\mathrm{fMRI}$ data. Neuroinformatics 7 : 37-53, 2009

27. Harden RN: Objectification of the diagnostic criteria for CRPS. Pain Med 11:1212-1215, 2010

28. Harden RN: The diagnosis of CRPS: Are we there yet? Pain 153:1142-1143, 2012

29. Harden RN, Bruehl S, Stanton-Hicks M, Wilson PR: Proposed new diagnostic criteria for complex regional pain syndrome. Pain Med 8:326-331, 2007

30. Hari R, Bourguignon M, Piitulainen H, Smeds E, De Tiège $\mathrm{X}$, Jousmäki V: Human primary motor cortex is both activated and stabilized during observation of other person's phasic motor actions. Philos Trans R Soc Lond B Biol Sci 369:20130171, 2014

31. Hotta J, Harno H, Nummenmaa L, Kalso E, Hari R, Forss N: Patients with complex regional pain syndrome overestimate applied force in observed hand actions. Eur J Pain 19: 1372-1381, 2015

32. Juottonen $\mathrm{K}$, Gockel M, Silén T, Hurri H, Hari R, Forss N: Altered central sensorimotor processing in patients with complex regional pain syndrome. Pain 98:315-323, 2002

33. Keysers C, Kaas JH, Gazzola V: Somatosensation in social perception. Nat Rev Neurosci 11:417-428, 2010

34. Kirveskari E, Vartiainen NV, Gockel M, Forss N: Motor cortex dysfunction in complex regional pain syndrome. Clin Neurophysiol 121:1085-1091, 2010

35. Klöppel S, Abdulkadir A, Jack CR, Koutsouleris $N$, Mourão-Miranda J, Vemuri P: Diagnostic neuroimaging across diseases. Neuroimage 61:457-463, 2012

36. Kriegeskorte N, Goebel R, Bandettini P: Informationbased functional brain mapping. Proc Natl Acad Sci U S A 103:3863-3868, 2006

37. Lamm C, Meltzoff AN, Decety J: How do we empathize with someone who is not like us? A functional magnetic resonance imaging study. J Cogn Neurosci 22: 362-376, 2010

38. Lewis JS, Kersten P, McPherson KM, Taylor GJ, Harris N, McCabe CS, Blake DR: Wherever is my arm? Impaired upper limb position accuracy in complex regional pain syndrome. Pain 149:463-469, 2010

39. Levy BJ, Wagner AD: Cognitive control and right ventrolateral prefrontal cortex: Reflexive reorienting, motor inhibition, and action updating. Ann N Y Acad Sci 1224:40-62. 2011

40. Maihöfner C, Baron R, DeCol R, Binder A, Birklein $F_{\imath}$ Deuschl G, Handwerker HO, Schattschneider J: The motor system shows adaptive changes in complex regional pain syndrome. Brain 130:2671-2687, 2007

41. Maihöfner C, Forster C, Birklein F, Neundörfer B, Handwerker $\mathrm{HO}$ : Brain processing during mechanical hyperalgesia in complex regional pain syndrome: A functional MRI study. Pain 114:93-103, 2005

42. Maihöfner $\mathrm{C}$, Handwerker HO, Birklein F: Functional imaging of allodynia in complex regional pain syndrome. Neurology 66:711-717, 2006

43. Mailis-Gagnon A, Nicholson K, Blumberger D, Zurowski M: Characteristics and period prevalence of selfinduced disorder in patients referred to a pain clinic with 
Hotta et al

the diagnosis of complex regional pain syndrome. Clin J Pain 24:176-185, 2008

44. Marinus J, Moseley GL, Birklein F, Baron R, Maihöfner C, Kingery WS, van Hilten JJ: Clinical features and pathophysiology of complex regional pain syndrome. Lancet Neurol 10: 637-648, 2011

45. Moseley GL, Birklein F, van Hilten JJ, Marinus J: Reply. Arthritis Care Res (Hoboken) 61:140-141, 2008

46. Moseley GL, Parsons TJ, Spence C: Visual distortion of a limb modulates the pain and swelling evoked by movement. Curr Biol 18:R1047-R1048, 2008

47. Moseley GL, Zalucki N, Birklein F, Marinus J, van Hilten JJ, Luomajoki H: Thinking about movement hurts: The effect of motor imagery on pain and swelling in people with chronic arm pain. Arthritis Rheum 59:623-631, 2008

48. Möttönen R, Järveläinen J, Sams M, Hari R: Viewing speech modulates activity in the left SI mouth cortex. Neuroimage 24:731-737, 2005

49. Nichols TE, Holmes AP: Nonparametric permutation tests for functional neuroimaging: A primer with examples. Hum Brain Mapp 15:1-25, 2002

50. Ochoa JL, Verdugo RJ: Neuropathic pain syndrome displayed by malingerers. J Neuropsychiatry Clin Neurosci 22: 278-286, 2010

51. Oertel BG, Preibisch C, Wallenhorst $T$, Hummel $T$, Geisslinger G, Lanfermann H, Lötsch J: Differential opioid action on sensory and affective cerebral pain processing. Clin Pharmacol Ther 83:577-588, 2008

52. Petrovic P, Kalso E, Petersson KM, Ingvar M: Placebo and opioid analgesia-imaging a shared neuronal network. Science 295:1737-1740, 2002

53. Ring D, Barth R, Barsky A: Evidence-based medicine: Disproportionate pain and disability. J Hand Surg Am 35: 1345-1347, 2010

54. Rizzolatti G, Fogassi L: The mirror mechanism: Recent findings and perspectives. Philos Trans R Soc Lond B Biol Sci 369:20130420, 2014

55. Sandroni $P$, Benrud-Larson LM, McClelland RL, Low PA: Complex regional pain syndrome type I: Incidence and prevalence in Olmsted county, a population-based study. Pain 103:199-207, 2003

56. Schwenkreis $\mathrm{P}$, Janssen $\mathrm{F}$, Rommel O, Pleger B, Volker $\mathrm{B}$, Hosbach I, Dertwinkel R, Maier C, Tegenthoff M: Bilateral motor cortex disinhibition in complex regional pain syndrome (CRPS) type I of the hand. Neurology 61:515-519, 2003

57. Schwoebel J, Friedman R, Duda N, Coslett HB: Pain and the body schema: Evidence for peripheral effects on mental representations of movement. Brain 124:2098-2104, 2001

58. Strafella AP, Paus T: Modulation of cortical excitability during action observation: A transcranial magnetic stimulation study. Neuroreport 11:2289-2292, 2000

59. Sundermann B, Burgmer M, Pogatzki-Zahn $E_{\text {, }}$ Gaubitz M, Stüber C, Wessolleck E, Heuft G, Pfleiderer B: Diagnostic classification based on functional connectivity in chronic pain: Model optimization in fibromyalgia and rheumatoid arthritis. Acad Radiol 21:369-377, 2014

60. Tzourio-Mazoyer N, Landeau B, Papathanassiou D, Crivello F, Etard O, Delcroix N, Mazoyer B, Joliot M: Automated anatomical labeling of activations in SPM using a macroscopic anatomical parcellation of the MNI MRI single-subject brain. Neuroimage 15:273-289, 2002

61. Ung $H$, Brown JE, Johnson KA, Younger J, Hush J, Mackey S: Multivariate classification of structural MRI data detects chronic low back pain. Cereb Cortex 24:1037-1044, 2014

62. Vapnik VN: The Nature of Statistical Learning Theory. New York, Springer-Verlag, 1995

63. Vartiainen N, Kirveskari E, Kallio-Laine K, Kalso E, Forss N: Cortical reorganization in primary somatosensory cortex in patients with unilateral chronic pain. J Pain 10: 854-859, 2009

64. Veldman PH, Reynen HM, Arntz IE, Goris RJ: Signs and symptoms of reflex sympathetic dystrophy: Prospective study of 829 patients. Lancet 342:1012-1016, 1993

65. Wager TD, Atlas LY, Lindquist MA, Roy M, Woo CW, Kross E: An fMRI-based neurologic signature of physical pain. N Engl J Med 368:1388-1397, 2013

66. Wager TD, Rilling JK, Smith EE, Sokolik A, Casey KL, Davidson RJ, Kosslyn SM, Rose RM, Cohen JD: Placeboinduced changes in FMRI in the anticipation and experience of pain. Science 303:1162-1167, 2004

67. Wiech K, Ploner M, Tracey I: Neurocognitive aspects of pain perception. Trends Cogn Sci 12:306-313, 2008

68. Yousry TA, Schmid UD, Alkadhi H, Schmidt D, Peraud A, Buettner A, Winkler P: Localization of the motor hand area to a knob on the precentral gyrus. A new landmark. Brain 120:141-157, 1997 